\title{
Research Report
}

\section{Pelvic Lymph Node Dissection may be Limited on the Contralateral Side in Strictly Unilateral Bladder Cancer without Compromising Oncological Radicality}

\author{
Bernhard Kiss ${ }^{1}$, Michael Paerli ${ }^{1}$, Daniel Schöndorf, Fiona C. Burkhard, George N. Thalmann \\ and Beat Roth* \\ Department of Urology, University of Bern, Bern, Switzerland
}

\begin{abstract}
.
Background: Results of a dynamic multimodality mapping study showed no lymphatic drainage of the lateral bladder wall to the contralateral internal iliac region.

Objectives: To validate whether pathoanatomical mapping in bladder cancer (BC) patients can confirm these results.

Methods: Between 01/2000 and 07/2013, 825 BC patients preoperatively staged $\geq$ pT1 and without clinical signs of metastases (cN0 cM0) underwent extended pelvic lymph node dissection (ePLND) and radical cystectomy at our department. Of these patients, 23\% (193/825) were lymph node (LN) positive in the pathological specimen; 26\% (51/193) of this subgroup had strictly unilateral BC. Pathoanatomical mapping was used to retrospectively validate the distribution of LN involvement in these 51 patients.

Results: A median of 35 LNs were removed per patient (range: 13-80 LNs), with a median of 2 positive LNs (range: 1-14 LNs). $27 \%$ (14/51) of patients presented with LN metastases on the contralateral side. No positive LNs were found in the contralateral internal iliac region or the contralateral fossa of Marcille. 10\% (5/51) of patients had LN metastases only on the contralateral side without evidence of metastases on the tumor-bearing side.

Conclusions: Our findings corroborate the data of a dynamic mapping study showing bilateral lymphatic drainage in almost one third of patients with strictly unilateral $\mathrm{BC}$, but no lymphatic drainage from the lateral bladder wall to the contralateral internal iliac region. If prospective studies confirm these results, the contralateral internal iliac region may be omitted during ePLND in patients with strictly unilateral BC.
\end{abstract}

Keywords: Bladder cancer, cystectomy, lymph node metastases, nerve sparing, unilateral tumor

\section{INTRODUCTION}

Two thirds of surgically treated patients with bladder cancer (BC) and lymph node (LN) metastases (pN+)

\footnotetext{
${ }^{1}$ These authors contributed equally to this work.

*Correspondence to: Beat Roth, Department of Urology, University of Bern, 3010 Bern, Switzerland. Tel.: +41316323641; Fax: +41316322181; E-mail: Urology.Berne@insel.ch.
}

will die from cancer [1]. Skinner has shown that 30\% of patients with only a few LN metastases can be cured by a meticulous $\mathrm{LN}$ dissection [2]. This suggests that in patients with invasive $\mathrm{BC}$ a bilateral extended pelvic lymph node dissection (ePLND) should be the minimal standard for optimal local and systemic oncologic control [3]. A recently published dynamic mapping study by Roth et al. [4] defined the standard PLND template as comprising the LNs along the common iliac 
artery up to where the ureter crosses the vessel, LNs above, below, and lateral to the external iliac artery and vein, the obturator fossa, and LNs medial and lateral to the internal iliac vessels. In fact, this template was already described by Whitmore and Marshall in 1962 [5]. In their dynamic mapping study, however, Roth et al. could show that almost half $(42 \%)$ of the internal iliac nodes are located medial to the hypogastric artery [4]. Dissection and removal of these LNs - called by some authors presacral LNs - bears the risk of harm to the autonomic nerves, threatening both continence and sexual function [6-12]. Since dynamic multimodality LN mapping has shown a drainage to the contralateral side in $40 \%$ of BC patients [4], a bilateral ePLND would appear to be mandatory in all $\mathrm{BC}$ patients, thus increasing the risk of negatively influencing autonomic nerve function. However, injection of radioactive tracer into the lateral bladder wall (non-tumor-bearing side) revealed no drainage to the contralateral internal iliac region [13]. We therefore used pathoanatomical mapping to validate the dynamic multimodality mapping findings suggesting that in strictly unilateral BC the contralateral internal iliac region may be omitted during PLND without compromising oncological radicality.

\section{MATERIAL AND METHODS}

A total of 825 BC patients preoperatively staged $\geq$ pT1 cN0 cM0 (physical examination, chest X-ray, abdominal and pelvic computerized tomography [CT] or magnetic resonance imaging [MRI], and bone scan) underwent ePLND and radical cystectomy at our institution between January 2000 and July 2013. Of these 825 patients, 193 (23\%) were found to have LN metastases upon pathological examination. None of these patients received neoadjuvant chemotherapy or neoadjuvant radiotherapy. Analysis of the localization of the primary tumor showed $51 \mathrm{pN}+$ patients to have strictly unilateral BC (lateral bladder wall) (Fig. 1, showing the institutional protocol of the anatomic tumor localization). The unilaterality was confirmed by reviewing the preoperative imaging (CT or MRI) of the pelvis and preoperative cystoscopy images/reports. It was confirmed by a review of pathology reports. All $51 \mathrm{pN}+$ patients had muscle-invasive disease $(\geq \mathrm{pT} 2)$, $49(96 \%)$ had poorly differentiated tumors (G3), and $28(55 \%)$ presented with concomitant carcinoma in situ not necessarily localized unilaterally (Table 1). Patients were followed according to our institutional standard post-cystectomy protocol 3, 6, 12, 18 and 24

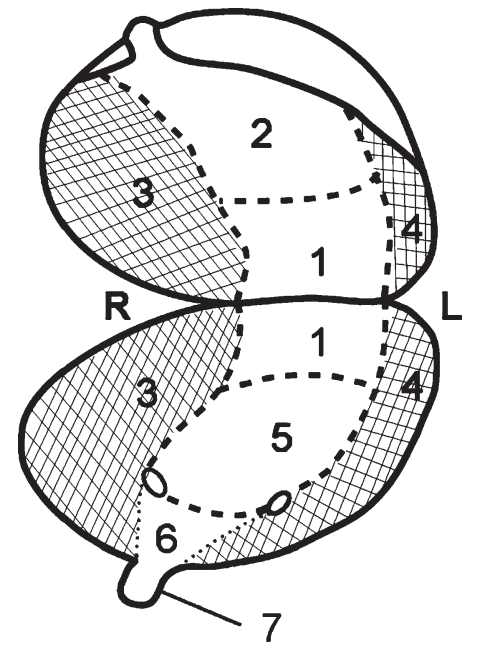

Fig. 1. Tumor localizations: (1) dome, (2) anterior wall, (3) right lateral wall, (4) left lateral wall, (5) posterior wall (6), bladder neck/trigone, (7) urethra. Only lymph node-positive bladder cancer patients with tumors strictly localized to the lateral wall (regions (3) and (4) in the figure) were included in the study.

Table 1

Patient characteristics and pathological features of 51 lymph nodepositive patients with strictly unilateral muscle-invasive bladder cancer

\begin{tabular}{lc}
\hline Sex; $n(\%)$ & \\
Male & $35(69)$ \\
Female & $16(31)$ \\
Age; median (range), y & $66.5(43-87)$ \\
Pathologic tumor stage; $n(\%)$ & \\
pT2a & $1(2)$ \\
pT2b & $6(12)$ \\
pT3a & $17(33)$ \\
pT3b & $20(39)$ \\
pT4 & $7(14)$ \\
Pathologic tumor grade; $n(\%)$ & \\
G2 & $2(4)$ \\
G3 & $49(96)$ \\
Concomitant carcinoma in situ; $n(\%)$ & \\
Absent & $23(45)$ \\
Present & $28(55)$ \\
Histological type; $n(\%)$ & \\
Squamous-cell carcinoma & $2(4)$ \\
Pure urothelial carcinoma & $33(65)$ \\
Variant histology in specimen & $16(31)$ \\
Squamous differentiation & $5(10)$ \\
Sarcomatoid differentiation & $3(6)$ \\
Other (nested, micropapillary, neuroendocrine) & $8(15)$ \\
Concomitant prostate cancer; $n(\%)$ & \\
Absent & $32(63)$ \\
Present & $19(37)$ \\
$\quad$ LN metastases of prostate cancer & $1(2)$ \\
\hline
\end{tabular}

months postoperatively, and yearly thereafter. CT scan and bone scan were performed 6, 12 and 24 months postoperatively. 


\section{Surgical technique}

Extended PLND started in all patients at the level of the crossing of the medialized ureter with the common iliac artery and continued distally to the iliac bifurcation (common iliac region) and along the external iliac vessels to the inguinal ligament to include the node of Cloquet bilaterally (external iliac region), as previously described [4]. The anterolateral border of the dissection field was the genito-femoral nerve. All tissue except the obturator nerve was removed from the obturator fossa (obturator region) and tissue removal was continued proximally along the obturator nerve and lateral to the iliac vessels to include the fossa of Marcille. Finally, the lymphatic tissue on the internal iliac vessels and their branches including the LNs medial to the internal iliac artery (internal iliac region) was removed. The excised tissue was fixed separately in neutral buffered formalin $(4 \%)$ and submitted in separate packages for pathological evaluation. The cystectomy protocol was reported in detail previously [14].

\section{Pathological analysis}

All specimens were processed and evaluated at the Institute of Pathology, University of Bern, Switzerland. Pathologists experienced in genitourinary pathology examined the cystectomy specimens and nodal tissues. No single pathologist examined all specimens but a common protocol was used. The bladder was filled with formalin and fixed at least overnight. Tumor characteristics noted were localization, size, extent of invasion, and multifocality. Sections for histology were taken from the tumor(s), including the site of deepest macroscopic invasion, the anterior, posterior and lateral walls of the bladder, the bladder neck with trigone, the dome of the bladder, and any macroscopically abnormal area of the mucosa (Fig. 1). Any residual ulcer after transurethral resection was sectioned in toto. Histologically, tumor type, tumor grade, tumor stage, the presence of vascular invasion, and carcinoma-in-situ were assessed. LNs were identified visually, by palpation, and by sectioning within each package after tissue had been degreased with acetone. All macroscopically detected LNs larger than $5 \mathrm{~mm}$ were cut into 2 -mm to 4 -mm-thick sections and completely embedded in paraffin. If no LNs could be identified, the entire tissue was embedded for histologic examination. One section per paraffin block was taken. The total number of LNs and the number bearing metastases were recorded for each site separately. Pathological staging was done according to the 1997
American Joint Committee on Cancer TNM classification.

\section{Statistical analysis}

Statistical analyses were performed using GraphPad Prism 5 Software (GraphPad, San Diego, CA). Local recurrences and distal metastases were compared using the chi-square test. Further results are purely descriptive due to the small patient sample size. Statistical significance was set at $p<0.05$.

\section{RESULTS}

A total of 1814 LNs (median 35 LNs; range: 13-80 LNs) were removed and examined in the $51 \mathrm{pN}+$ patients with strictly unilateral BC (Table 2). Of these 1814 LNs, 145 (8\%) showed metastases upon pathological examination. The distribution of all positive LNs is shown in Fig. 2. The median number of positive LNs per patient was 2 (range: $1-14$ ). Twenty-five of the 145 positive LNs (17\%) showed extracapsular extension of the LN metastasis. Fourteen of the 51 $\mathrm{pN}+$ patients $(27 \%)$ presented with LN metastases on the contralateral side (cross-over). The total number of positive contralateral LNs was 18 (median $1 \mathrm{LN}$ per patient; range: $1-3)$. Of the 18 positive contralateral LNs, $13(72 \%)$ were located in the external iliac (region I), 3 (17\%) in the obturator fossa (region II), and $2(11 \%)$ in the common iliac region (region IV). Not a single LN metastasis was found in the contralateral internal iliac region (region III) or the contralateral fossa of Marcille (region V) (Fig. 2). Five of the 51 $\mathrm{pN}+$ patients $(10 \%)$ showed LN metastases only in contralateral LNs without metastases to LNs in the ipsilateral pelvis (total LNs: 7; external iliac: 3 [42\%]; common iliac: 1 [14\%]; obturator fossa: 3 [42\%]).

Table 2

Distribution of the 1814 LNs resected in 51 patients

\begin{tabular}{lcccc}
\hline & $n$ & $\%$ & median & IQR \\
\hline Ipsilateral & 966 & 53 & & \\
- external iliac & 454 & 25 & 7 & $4.5-12$ \\
- obturator fossa & 218 & 12 & 4 & $2-6$ \\
- internal iliac & 145 & 8 & 3 & $1-5$ \\
- common iliac & 99 & 5 & 2 & $1-4$ \\
- fossa of Marcille & 50 & 3 & 1 & $0-3$ \\
Contralateral & 848 & 47 & & \\
- external iliac & 460 & 25 & 8 & $4-12$ \\
- obturator fossa & 197 & 12 & 4 & $2-5$ \\
- internal iliac & 106 & 6 & 2.5 & $1-4$ \\
- common iliac & 62 & 3 & 1.5 & $0-4$ \\
- fossa of Marcille & 23 & 1 & 0.5 & $0-1.5$ \\
IQR: Interquartile range & & & & \\
\hline
\end{tabular}




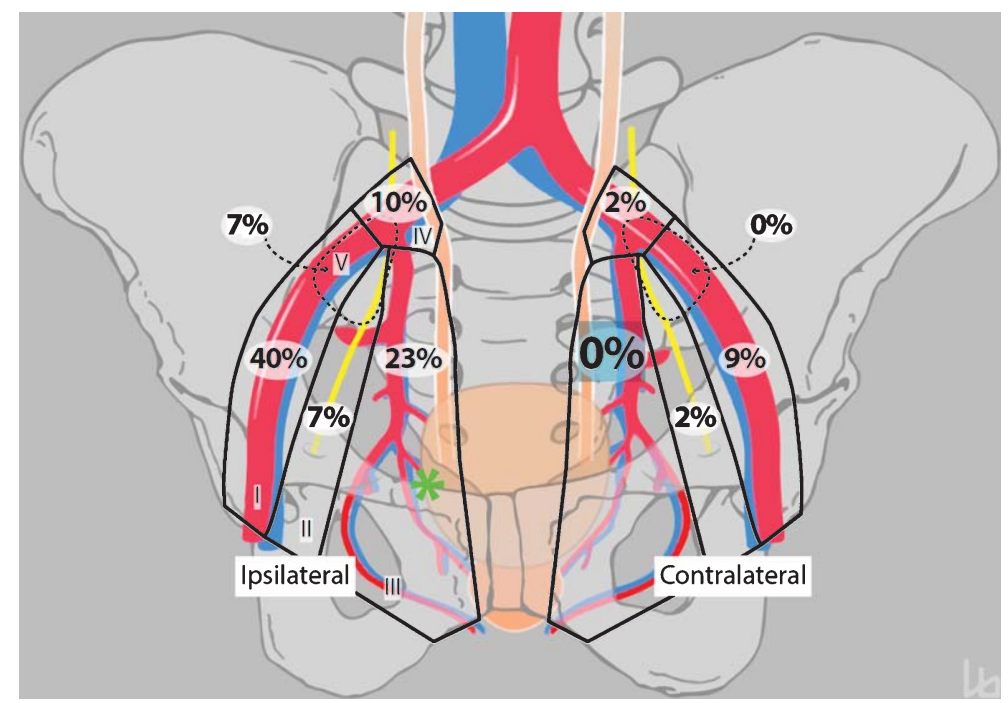

\begin{tabular}{|l|c|c|c|c|c|}
\hline & $\begin{array}{c}\text { external } \\
\text { iliac }\end{array}$ & $\begin{array}{c}\text { obturator } \\
\text { fossa }\end{array}$ & $\begin{array}{c}\text { internal } \\
\text { iliac }\end{array}$ & $\begin{array}{c}\text { common } \\
\text { iliac }\end{array}$ & $\begin{array}{c}\text { fossa of } \\
\text { Marcille }\end{array}$ \\
\hline ipsilateral $(n)$ & 58 & 10 & 33 & 15 & 10 \\
\hline contralateral $(n)$ & 13 & 3 & 0 & 3 & 0 \\
\hline
\end{tabular}

Fig. 2. Schematic diagram showing the distribution of lymph node metastases $(n=145)$ of the lateral bladder walls (left and right) projected to a single side (right; asterisk). The pelvic lymph node dissection boundaries are subdivided into the (I) external iliac, (II) obturator fossa, (III) internal iliac, (IV) common iliac, and (V) fossa of Marcille regions.

One of the $51 \mathrm{pN}+$ patients with strictly unilateral $\mathrm{BC}(2 \%)$ developed a local recurrence during follow up versus 5 of $142 \mathrm{pN}+$ patients with $\mathrm{BC}$ localization other than strictly unilateral $(3.5 \% ; p=0.58)$; distant metastases were seen in 10 of $51(20 \%)$ of the former patients and 30 of $142(21 \%)$ of the latter $(p=0.82)$.

\section{DISCUSSION}

Extended PLND and radical cystectomy are the gold standard in the management of muscle-invasive BC. More and more data is being gathered confirming the benefit of a meticulous PLND in terms of recurrencefree survival and overall survival $[3,15,16]$. Still, there is controversy regarding the optimal extent of the PLND template - especially the proximal border. In their comparative cystectomy series, Zehnder et al. [3] showed similar survival and recurrence rates in pT2-3 cN0 cM0 BC patients receiving a meticulous ePLND up to the mid upper third of the common iliac vessel as compared to a super-extended template up to the inferior mesenteric artery. This was the first time it could be shown that more is not always better in PLND for BC. In line with these considerations and the results of a dynamic mapping study of Roth et al. [4], our pathoanatomical validation study corroborates the hypothesis that there is no lymphatic drainage to the contralateral internal iliac region in patients with strictly unilateral $\mathrm{BC}$. To confirm that PLND of the contralateral internal iliac region can be safely omitted in these patients, however, would require multi-center validation. Preferably, this should be done in a prospective protocol evaluating the survival of patients with strictly unilateral tumors who undergo ePLND versus patients who undergo ePLND that excludes the contralateral internal iliac region. Alternatively, a prospective multi-center study could be conducted with careful documentation of locations of the primary tumor (bladder mapping) and of nodal metastases to evaluate the true incidence and location of ipsilateral and contralateral metastases.

To date little attention has been paid to the relation of the primary tumor site within the bladder to the site of metastatic deposit. Literature on intravesical tumor location and oncological parameters is sparse. Moreover, the available data are mutually conflicting [10, $17,18]$. In addition none of the published trials investigated the primary lymphatic landing sites with regard to tumor location. More recently, Svatek et al. found 
that tumor involvement of the trigone is associated with a greater risk of LN metastases and an unfavorable cancer-specific survival, but no other tumor location (especially the lateral wall) was found to be associated with an increased risk of LN involvement [19]. In our cohort, however, local recurrence and distal metastasis rates in $\mathrm{pN}+$ patients with strictly unilateral $\mathrm{BC}$ did not differ significantly from those in $\mathrm{pN}+$ patients with other BC locations ( $2 \%$ vs. $3.5 \%, p=0.58 ; 20 \%$ and $21 \%, p=0.82$, respectively).

Crossover LN metastases was found in $27 \%$ of our $\mathrm{pN}+$ patients with strictly unilaterally localized BC. This finding accords with earlier pathoanatomical studies $[10,20]$ showing contralateral LN metastases in $15.5 \%$ to $41 \%$ of patients with unilateral BC. Most importantly, $10 \%(5 / 51)$ of our $\mathrm{pN}+$ patients with unilateral $\mathrm{BC}$ had $\mathrm{LN}$ metastases on the contralateral side only. This number is lower than the 23\% (3/13) of patients with unilateral BC in the study of Leissner et al. [20]. Indeed, they included not only strictly unilateral tumors but tumors localized in a single bladder hemisphere, thus most probably including tumors involving the bladder neck, trigone, and/or posterior bladder wall - regions within the bladder that are well known to have ubiquitous lymphatic drainage [4, 19]. The finding that $27 \%$ of our patients had bilateral and $10 \%$ had contralateral metastases only shows once again that bilateral PLND is an oncological cornerstone of surgery for BC.

This study has limitations beyond the inherent shortcomings of any retrospective study. Although our pathoanatomical study examines a relatively large cohort of $\mathrm{pN}+\mathrm{BC}$ patients $(n=193)$, the sample size of $51 \mathrm{pN}+$ patients with strictly unilateral $\mathrm{BC}$ is relatively small. Still, our data corroborate the finding of a dynamic LN mapping study that lymphatic drainage to the contralateral internal iliac region is absent in strictly unilateral tumors [4]; there was not a single $\mathrm{LN}$ metastasis in the contralateral internal iliac region, not even in patients with high tumor stage / grade and high LN metastatic burden. This appears to contrast with the finding by Leissner et al. of some positive nodes in the contralateral internal iliac region [20]. Indeed, in that study they examined tumors located in a single bladder hemisphere including tumors involving the bladder neck, trigone, and/or posterior bladder wall, regions well known to drain everywhere within the pelvis [4, 19].

Another limitation of the present study is that no prospective or retrospective data on LN sparing in the internal iliac region are yet available. Nevertheless, the possible functional benefits are a logical conclusion based on anatomical and functional studies. The branches of the pelvic plexus (comprised of sympathetic and parasympathetic fibres) [21] are in close proximity to the (medial) internal iliac region. Omission of LN dissection in this internal iliac region (on the side contralateral to the tumor) should minimize damage to the fragile autonomic nerves which appear to be important for innervation of the proximal urethra (continence) [22, 23]; such nerve sparing may have a beneficial impact on continence after radical cystoprostatectomy and ileal orthotopic bladder substitution $[8,24,25]$ although there is no direct evidence for this yet. These clinical data accord with intraoperative electrophysiological findings and animal studies [12, 26]. Moreover, nerve sparing also appears to safeguard erectile function by preserving parasympathetic fibres passing through the plexus pelvicus [6-8, 11, 27-29]. It is, however, not only the preservation of the neurovascular bundle that makes the omission of LN dissection in the (medial) internal iliac region appealing for the improvement of postoperative functional results, but also the potential further decrease in devascularization (damage to internal iliac vessel branches) in this region with concomitant less ischemia and necrosis [11]. Nerve sparing is of utmost importance not only in terms of better functional results of subsequent orthotopic bladder substitution or better recovery of sexual function, but also as a means to avert potential problems of defecation [30]. As the present results indicate, this may be done without compromising oncological radicality. Prospective evaluation (preferably multicenter), however, has to confirm this hypothesis.

Another limitation of this study is the difficulty with assigning tumor location in a retrospective study. We did, however, look not only at pathology reports but also at (preoperative) cystoscopy reports. Furthermore, imaging data were all re-reviewed. Therefore, we think that the risk of "mis-assignment" of tumor locations was minimal.

In conclusion, our pathoanatomical validation study corroborates the data of a dynamic mapping study showing a lack of lymphatic drainage to the contralateral internal iliac region in patients with strictly unilateral BC. If these results can be confirmed in a prospective evaluation - preferably a multi-center study - contralateral pelvic lymph node dissection could then be limited to the obturator fossa and the external and common iliac regions in this highly selected group of patients with strictly unilateral tumor growth. This would better preserve the contralateral autonomic nerves situated close and medial to the internal iliac vessels. 


\section{ACKNOWLEDGMENTS}

None.

\section{CONFLICT OF INTEREST}

The authors have no conflict of interest to report.

\section{REFERENCES}

[1] Stein JP, Lieskovsky G, Cote R, Groshen S, Feng AC, Boyd S, Skinner E, Bochner B, Thangathurai D, Mikhail M, Raghavan $\mathrm{D}$, Skinner DG. Radical cystectomy in the treatment of invasive bladder cancer: Long-term results in 1,054 patients. Journal of Clinical Oncology : Official Journal of the American Society of Clinical Oncology 2001;19(3):666-75.

[2] Skinner DG. Management of invasive bladder cancer: A meticulous pelvic node dissection can make a difference. The Journal of Urology 1982;128(1):34-6.

[3] Zehnder P, Studer UE, Skinner EC, Dorin RP, Cai J, Roth B, Miranda G, Birkhäuser F, Stein J, Burkhard FC, Daneshmand S, Thalmann GN, Gill IS, Skinner DG. Super extended versus extended pelvic lymph node dissection in patients undergoing radical cystectomy for bladder cancer: A comparative study. The Journal of Urology 2011;186(4):1261-8.

[4] Roth B, Wissmeyer MP, Zehnder P, Birkhäuser FD, Thalmann GN, Krause TM, Studer UE. A new multimodality technique accurately maps the primary lymphatic landing sites of the bladder. European Urology 2010;57(2):205-11.

[5] Whitmore WF, Jr., Marshall VF. Radical total cystectomy for cancer of the bladder: 230 consecutive cases five years later. The Journal of Urology 1962;87:853-68.

[6] Bhatt A, Nandipati K, Dhar N, Ulchaker J, Jones S, Rackley R, Zippe C. Neurovascular preservation in orthotopic cystectomy: Impact on female sexual function. Urology 2006;67(4):742-5.

[7] Hekal IA, El-Bahnasawy MS, Mosbah A, El-Assmy A, Shaaban A. Recoverability of erectile function in post-radical cystectomy patients: Subjective and objective evaluations. European Urology 2009;55(2):275-83.

[8] Kessler TM, Burkhard FC, Perimenis P, Danuser H, Thalmann GN, Hochreiter WW, Studer UE. Attempted nerve sparing surgery and age have a significant effect on urinary continence and erectile function after radical cystoprostatectomy and ileal orthotopic bladder substitution. The Journal of Urology 2004;172(4 Pt 1):1323-7.

[9] Madersbacher S, Studer UE. Contemporary cystectomy and urinary diversion. World Journal of Urology 2002;20(3): 151-7.

[10] Mills RD, Turner WH, Fleischmann A, Markwalder R, Thalmann GN, Studer UE. Pelvic lymph node metastases from bladder cancer: Outcome in 83 patients after radical cystectomy and pelvic lymphadenectomy. The Journal of Urology 2001;166(1):19-23.

[11] Stenzl A. Pelvic neuroanatomy and recovery of potency. European Urology 2009;55(2):284-6.

[12] Strasser H, Ninkovic M, Hess M, Bartsch G, Stenzl A. Anatomic and functional studies of the male and female urethral sphincter. World Journal of Urology 2000;18(5):324-9.
[13] Roth B, Zehnder P, Birkhauser FD, Burkhard FC, Thalmann GN, Studer UE. Is bilateral extended pelvic lymphadenectomy necessary for strictly unilateral invasive bladder cancer? The Journal of Urology 2012;187(5):1577-82.

[14] Studer UE, Danuser H, Merz VW, Springer JP, Zingg EJ. Experience in 100 patients with an ileal low pressure bladder substitute combined with an afferent tubular isoperistaltic segment. The Journal of Urology 1995;154(1):49-56.

[15] Dhar NB, Klein EA, Reuther AM, Thalmann GN, Madersbacher S, Studer UE. Outcome after radical cystectomy with limited or extended pelvic lymph node dissection. The Journal of Urology 2008;179(3):873-8.

[16] Stenzl A, Cowan NC, De Santis M, Kuczyk MA, Merseburger AS, Ribal MJ, Sherif A, Witjes JA. Treatment of muscleinvasive and metastatic bladder cancer: Update of the EAU guidelines. European Urology 2011;59(6):1009-18.

[17] Martin M, Bernardini S, Kleinclauss F, Della Negra E, Henry $\mathrm{PC}$, Bittard H. Prognostic value of tumor location of urothelial tumors of the bladder, after total cystectomy. Progres en urologie : Journal de l'Association francaise d'urologie et de la Societe francaise d'urologie 2002;12(6):1221-7.

[18] Wedel SA, Jones J, Tsaur I, Ackermann H, Beecken WD, Jonas D, Blaheta R. Association of intravesical tumor location with metastases to the pelvic lymph nodes in transitional cell cancer of the bladder. The American Journal of the Medical Sciences 2010;339(4):341-4.

[19] Svatek RS, Clinton TN, Wilson CA, Kamat AM, Grossman HB, Dinney CP, Shah JB. Intravesical tumor involvement of the trigone is associated with nodal metastasis in patients undergoing radical cystectomy. Urology 2014;84(5): 1147-51.

[20] Leissner J, Ghoneim MA, Abol-Enein H, Thüroff JW, Franzaring L, Fisch M, Schulze H, Managadze G, Allhoff EP, el-Baz MA, Kastendieck H, Buhtz P, Kropf S, Hohenfellner R, Wolf HK. Extended radical lymphadenectomy in patients with urothelial bladder cancer: Results of a prospective multicenter study. The Journal of Urology 2004;171(1):139-44.

[21] Baader B, Herrmann M. Topography of the pelvic autonomic nervous system and its potential impact on surgical intervention in the pelvis. Clin Anat 2003;16(2):119-30.

[22] Alsaid B, Bessede T, Diallo D, Moszkowicz D, Karam I, Benoit G, Droupy S. Division of autonomic nerves within the neurovascular bundles distally into corpora cavernosa and corpus spongiosum components: Immunohistochemical confirmation with three-dimensional reconstruction. European Urology 2011;59(6):902-9.

[23] Kaiho Y, Nakagawa H, Saito H, Ito A, Ishidoya S, Saito S Arai Y. Nerves at the ventral prostatic capsule contribute to erectile function: Initial electrophysiological assessment in humans. European Urology 2009;55(1):148-54.

[24] Hautmann RE. Urinary diversion: Ileal conduit to neobladder. The Journal of Urology 2003;169(3):834-42.

[25] Turner WH, Danuser H, Moehrle K, Studer UE. The effect of nerve sparing cystectomy technique on postoperative continence after orthotopic bladder substitution. The Journal of Urology 1997;158(6):2118-22.

[26] Nelson CP, Montie JE, McGuire EJ, Wedemeyer G, Wei JT. Intraoperative nerve stimulation with measurement of urethral sphincter pressure changes during radical retropubic prostatectomy: A feasibility study. The Journal of Urology 2003;169(6):2225-8. 
[27] Eichelberg C, Erbersdobler A, Michl U, Schlomm T, Salomon G, Graefen M, Huland H. Nerve distribution along the prostatic capsule. European Urology 2007;51(1):105-10; discussion 10-1.

[28] Sievert KD, Hennenlotter J, Laible I, Amend B, Schilling $D$, Anastasiadis A, Kuehs U, Nagele U, Stenzl A. The periprostatic autonomic nerves-bundle or layer? European Urology 2008;54(5):1109-16.
[29] Walsh PC, Donker PJ. Impotence following radical prostatectomy: Insight into etiology and prevention. The Journal of Urology 1982;128(3):492-7.

[30] Rao GN, Drew PJ, Lee PW, Monson JR, Duthie GS. Anterior resection syndrome is secondary to sympathetic denervation. International Journal of Colorectal Disease 1996;11(5):250-8. 